\title{
Dynamic Characteristics Analysis with Multi-Directional Coupling in a TBM Mainframe
}

\author{
Laikuang Lin ${ }^{1,2}$, Yimin Xia ${ }^{1,2^{*}}$, Zhengguang Li ${ }^{1}$, Caizhang Wu${ }^{1}$, Yongliang Cheng ${ }^{1,3}$ and Qing Tan ${ }^{1,2}$
}

\begin{abstract}
The cutterhead of a full-face rock tunnel boring machine (TBM) is constantly subjected to varying impact and dynamic loads during tunneling processes, resulting in relatively large vibrations that could easily lead to fatigue cracking of the entire machine and affect the tunneling performance and efficiency. To explore the dynamic characteristics of the TBM mainframe, a TBM from a water-diversion project is investigated in this research. According to the TBM vibration transmission route, an equivalent dynamic model of the TBM mainframe is established using the lumped-mass method in which the relevant dynamic parameters are solved. Additionally, the dynamic response characteristics of the TBM mainframe are analyzed. The results indicate that the vibration levels in three directions are approximately the same, the multi-directional vibration of the cutterhead is more intense than that of other components, and the vibration and external excitation exhibit identical change trends. A set of vibration field tests is performed to analyze the in situ dynamic responses of the mainframe and verify the correctness of the dynamic model. The theoretical and measured acceleration values of the TBM mainframe have the same magnitude, which proves the validity of the dynamic model and its solution. The aforementioned results provide an important theoretical value and practical significance for the design and assessment of the TBM mainframe.
\end{abstract}

Keywords: Tunnel boring machine, Time-varying excitation, Dynamic model, Load transfer, Dynamic response

\section{Introduction}

The tunnel boring machine (TBM) is large-scale underground equipment that is widely used in underground tunnel projects owing to high safety and reliability, low manpower requirement, minor environmental damages, and rapid excavation speed [1,2]. As a key component of the TBM, the mainframe is composed of a cutterhead system, main drive system, shield, and main girder as well as a supporting-thrust system. Because of complicated geological conditions and variable tunneling parameters, a tunnel often features high strength and high confining pressure as well as a high quartz content, along with the step crushing of rocks. In the tunneling process, the TBM cutterhead is subjected to large thrust and torque caused

\footnotetext{
*Correspondence: xiaymj@csu.edu.cn

${ }^{1}$ College of Mechanical and Electrical Engineering, Central South University, Changsha 410083, China

Full list of author information is available at the end of the article
}

by the interaction between the cutters and rock [3, 4], which may result in significant damages, such as cracking of the cutterhead and loosening or separation of bolts, and affect the fatigue life of the TBM mainframe.

Studies on the TBM mainframe system have been carried out by several researchers, including those on external loads, thrust, torque, overturning torque, and unbalanced radial force in the tunneling process. Relevant models have also been established to conduct dynamic characteristic analyses of the cutterhead system. Rostami [5] analyzed various factors affecting the rock-breaking load of a disc cutter, established the CSM (Colorado School of Mines) force-estimation model for the cutter, and evaluated the performance of the cutterhead from the perspective of disc cutter layouts. Xia et al. [6] studied the formation mechanism and change pattern of the side force suffered by the center cutter and set up a calculation model for predicting the average side force. Huo et al. [7] subdivided the TBM disc cutter into center, inner, and gauge cutters and 
presented a multi-stage rock fragmentation load prediction model for disc cutter groups based on the dense-core theory and types of disc cutters. Shi et al. [8] put forward a method for calculating the cutterhead torque by taking into account the cutterhead structure, cutting principle, and geological conditions. Additionally, Zhou et al. [9] and Geng et al. [10] studied the factors that influence the thrust and torque of cutterheads and derived a model for calculating the relevant thrust, torque, overturning torque, and other external loads. Xia et al. [11] modified the cutterhead overturning moment calculation model and analyzed the mechanical performance of a typical TBM cutterhead under different working conditions. Zhao et al. [12] analyzed the composition of earth pressure balance (EPB) TBM cutterhead torque and developed a theoretical TBM torque model for a rock-soil interface mixed ground. Because of the difficulties in obtaining the accurate load of the cutterhead using theoretical analysis, test methods have been applied to acquire the load data of the cutterhead and its change trends. A test of external loads was performed by Entacher et al. [13, 14], who presented a method to detect the three-directional loading of a disc cutter in real-time and conducted a field test in the Austrian Koralm Tunnel. Geng et al. [15] proposed an experimental method to investigate the rock-cutting process of a TBM gauge cutter. According to the theoretical models, Zhang et al. [16, 17] analyzed the total load in an EPB TBM tunneling process and established a predictive model for the total load, which reflected the influence of the geological, operating, and structural parameters. Sun et al. $[18,19]$ and Li et al. [20] established a coupling nonlinear dynamic model of a cutterhead system to analyze the dynamic characteristics of the cutterhead. Huo et al. [21, 22] established multi-degreeof-freedom coupling dynamic models for the disc cutter and cutter system and revealed the dynamic characteristics of the disc cutter and cutter system. In addition, Huo et al. [23, 24] presented multi-coupling dynamic models for the TBM main drive and supporting systems and carried out a field strain test to verify the models. Festa et al. [25] set up a kinematic model for a TBM based on theoretical and geometrical considerations, and the ground displacements were obtained through the model and verified using the TBM monitoring data. In addition, considering the redundant driven rotary system, propulsion system, and geological conditions, Zhang et al. [26] and Huang et al. [27] established a mutual coupling dynamic model for cutterhead tunneling to analyze the dynamic characteristics of the rotary and supporting-propulsion systems under complex geological conditions.

As mentioned before, efforts have been intensified in the research and design of TBM cutterheads. However, profound studies on the TBM mainframe from the perspective of dynamics have not yet been performed. The existing dynamic model has been simplified to merely analyze the data of the main drive without considering the supporting or propelling forces provided by the main girder and gripper shoes in the mainframe. As a result, the transmission path is not closed. Additionally, differences in the frequency spectrum characteristics exist between the simulation-predicted dynamic and real external loads. Therefore, to investigate the dynamic characteristics of the TBM mainframe, a dynamic model of a TBM mainframe is established, which comprehensively considers the time-varying external excitations, transmission route of the vibration, and three-dimensional forces obtained from rock-breaking experiments. In addition, a set of field tests is carried out to collect field data and validate the correctness of the dynamic model. The present study provides a theoretical basis for evaluating and designing the TBM mainframe structure that can effectively prevent cracking, reduce the vibrations of the TBM mainframe, and prolong the fatigue life of the main bearing as well as improve tunneling efficiency.

\section{Dynamic Model of TBM Mainframe}

During the tunneling process, the vibrations of the TBM mainframe consist of lateral, vertical, tunneling, lateral overturning, and vertical overturning vibrations. The loads acting on the mainframe are shown in Figure 1, where $F_{z}$ and $T$ are the thrust force in the tunneling direction and total torque, respectively, and $M, F_{x}$, and $F_{y}$ represent the overturning moment, lateral unbalanced force, and vertical unbalanced force, respectively. Total thrust $F_{t}$ is provided by the propelling hydraulic cylinders to move the TBM forward. The cutterhead, main drive, and first section of the main girder are mainly backed by the bottom shield, which are tightly attached to the rock, thus bearing the friction resistance force $F_{f}$ caused by gravity $G$.

The transmission path of the mainframe external excitations and vibrations is opposite that of the TBM




tunneling thrust. The three-directional impact loads of the cutterhead are created by the disc cutters that break the rock during TBM tunneling. Through a connection flange in the cutterhead, the load and vibration are transmitted from the cutterhead to the main bearing and subsequently to the other parts behind the main drive. Then, through the main girder and propelling hydraulic cylinders at both sides of the main girder, the load and vibration are transmitted to the saddle holder of the gripper shoes that grip the rock, providing a forward reaction force and reducing the vibration of the gripper shoes.

According to the analysis of the load transmission path in the TBM mainframe, the lumped-mass method is adopted to simplify the connection relationships of each component to the spring-damping system to establish an equivalent dynamic model of the TBM mainframe, as shown in Figures 2 and 3.

Figure 2 shows a dynamic model of the lateral and vertical vibrations of the mainframe. Figure 3 shows a dynamic model that represents the coupling relationship between the tunneling and overturning vibrations in the mainframe. When the overturning vibration is calculated, the connection relationships between the cutterhead and the main bearing are simplified into four spring-damping systems. The whole overturning stiffness of the cutterhead is equal to the tunneling joint stiffness. In the dynamic model, $x, y$, and $z$ denote the lateral, vertical, and tunneling directions, respectively. $m_{1}, m_{2}, m_{3}, m_{41}, m_{42}, m_{43}$, and $m_{5}$ represent the masses of the cutterhead, main bearing, main drive (including the shield), main girder 1, main girder 2, main



Figure 2 Dynamic model of the lateral and vertical vibrations of the TBM mainframe

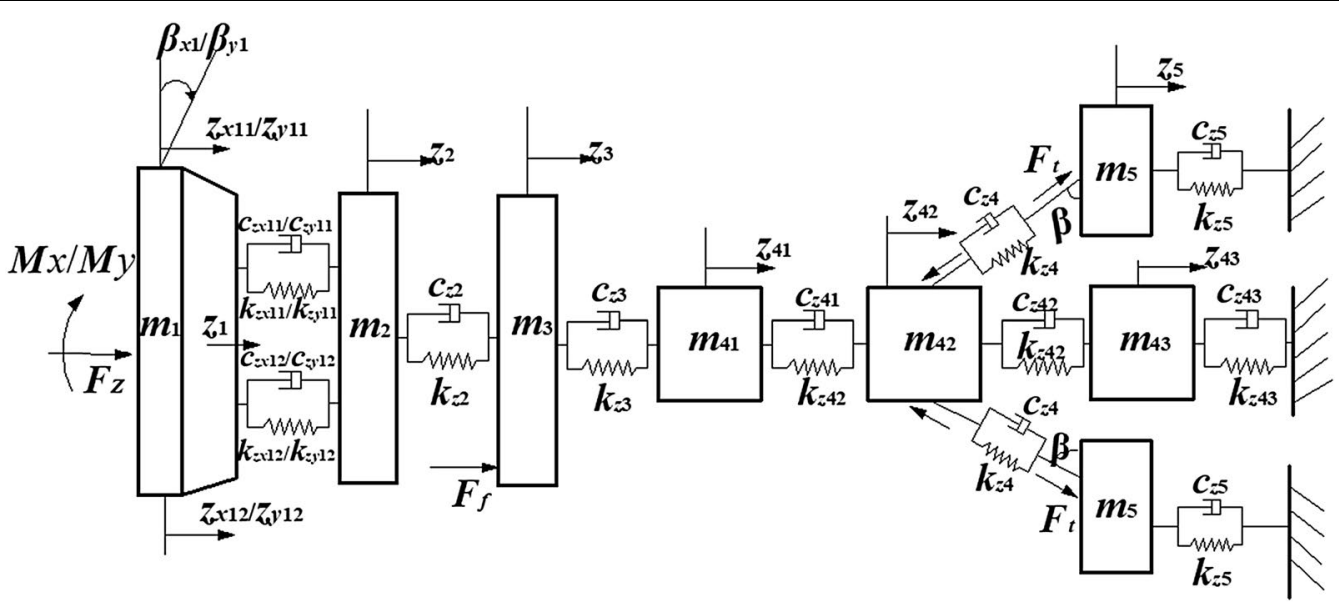

Figure 3 Coupling dynamic model of the tunneling and overturning vibrations of the TBM mainframe 
girder 3 , and gripper shoes, respectively. $x_{1}, x_{2}, x_{3}, x_{41}, x_{42}$, $x_{43}$, and $x_{5}$ represent the lateral degrees of freedom of the cutterhead, main bearing, main drive, main girder 1 , main girder 2, main girder 3 , and gripper shoes respectively. $y$, $z$, and $\theta$ denote the corresponding vertical, tunneling, and overturning degrees of freedom respectively. $k_{x 1}, k_{x 2}, k_{x 3}$, $k_{x 41}, k_{x 42}, k_{x 43}, k_{x 5}, k_{y 1}, k_{y 2}, k_{y 3}, k_{y 41}, k_{y 42}, k_{y 43}$, and $k_{y 5}$ denote the lateral and vertical equivalent stiffness values of the cutterhead, main bearing, main drive, main girder 1 , main girder 2, main girder 3 , and gripper shoes, whereas $c$ represents the corresponding damping.

According to the dynamic model, the dynamic equations of the vibration transmission in the TBM mainframe under different degrees of freedom are presented. The lateral and vertical degrees of freedom are expressed as follows: where $I_{1 x}$ and $I_{1 y}$ are the lateral and vertical moments of inertia, respectively. $k_{z x 11}, k_{z x 12}, k_{z y 11}$, and $k_{z y 12}$ are the tunneling equivalent stiffness values of the four pieces of cutterhead.

Coupling relationships exist between the tunneling and overturning vibrations. The relationships between the displacement of each cutterhead pieces and that of the cutterhead tunneling vibration are expressed in Eq. (4):

$$
\left\{\begin{array}{l}
z_{x 11}=z_{1}-a_{L} \sin \theta_{x 1} \\
z_{x 12}=z_{1}+a_{L} \sin \theta_{x 1} \\
z_{y 11}=z_{1}-a_{L} \sin \theta_{y 1} \\
z_{y 12}=z_{1}+a_{L} \sin \theta_{y 1}
\end{array}\right.
$$

The tunneling degree of freedom is expressed as follows:

$$
\left\{\begin{array}{l}
m_{1} \cdot \ddot{z}_{1}+c_{z x 11}\left(\dot{z}_{x 11}-\dot{z}_{2}\right)+c_{z x 12}\left(\dot{z}_{x 12}-\dot{z}_{2}\right)+c_{z y 11}\left(\dot{z}_{y 11}-\dot{z}_{2}\right)+c_{z y 12}\left(\dot{z}_{y 12}-\dot{z}_{2}\right) \\
\quad+k_{z x 11}\left(z_{x 11}-z_{2}\right)+k_{z x 12}\left(z_{x 12}-z_{2}\right)+k_{z y 11}\left(z_{y 11}-z_{2}\right)+k_{z y 12}\left(z_{y 12}-z_{2}\right)=F_{z} \\
m_{2} \cdot \ddot{z}_{2}+c_{z x 11}\left(\dot{z}_{2}-\dot{z}_{x 11}\right)+c_{z x 12}\left(\dot{z}_{2}-\dot{z}_{x 12}\right)+c_{z y 11}\left(\dot{z}_{2}-\dot{z}_{y 11}\right)+c_{z y 12}\left(\dot{z}_{2}-\dot{z}_{y 12}\right)+c_{z 2}\left(\dot{z}_{2}-\dot{z}_{3}\right) \\
\quad+k_{z x 11}\left(z_{2}-z_{x 11}\right)+k_{z x 12}\left(z_{2}-z_{x 12}\right)+k_{z y 11}\left(z_{2}-z_{y 11}\right)+k_{z y 12}\left(z_{2}-z_{y 12}\right)+k_{z 2}\left(z_{2}-z_{3}\right)=0 \\
m_{3} \cdot \ddot{z}_{3}+c_{z 2}\left(\dot{z}_{3}-\dot{z}_{2}\right)+c_{z 3}\left(\dot{z}_{3}-\dot{z}_{41}\right)+k_{z 2}\left(z_{3}-z_{2}\right)+k_{z 3}\left(z_{3}-z_{41}\right)=F_{f} \\
m_{41} \cdot \ddot{z}_{41}+c_{z 3}\left(\dot{z}_{41}-\dot{z}_{3}\right)+c_{z 41}\left(\dot{z}_{41}-\dot{z}_{42}\right)+k_{z 3}\left(z_{41}-z_{3}\right)+k_{z 41}\left(z_{41}-z_{42}\right)=0 \\
m_{42} \cdot \ddot{z}_{42}+c_{z 41}\left(\dot{z}_{42}-\dot{z}_{41}\right)+c_{z 42}\left(\dot{z}_{42}-\dot{z}_{43}\right)+2 c_{z 4}\left(\dot{z}_{42}-\dot{z}_{5}\right) \\
\quad+k_{z 41}\left(z_{42}-z_{41}\right)+k_{z 42}\left(z_{42}-z_{43}\right)+2 k_{z 4}\left(z_{42}-z_{5}\right)=-2 F_{t} \\
m_{43} \cdot \ddot{z}_{43}+c_{z 42}\left(\dot{z}_{43}-\dot{z}_{42}\right)+c_{z 43} \cdot \dot{z}_{43}+k_{z 42}\left(z_{43}-z_{42}\right)+k_{z 43} \cdot z_{43}=0 \\
m_{5} \cdot \ddot{z}_{5}+c_{z 4}\left(\dot{z}_{5}-\dot{z}_{42}\right)+c_{z 5} \cdot \dot{z}_{5}+k_{z 4}\left(z_{5}-z_{42}\right)+k_{z 5} \cdot z_{5}=F_{t} .
\end{array}\right.
$$

The overturning degree of freedom is expressed as follows:

$$
\left\{\begin{array}{c}
I_{1 x} \cdot \ddot{\theta}_{x 1}-c_{z x 11}\left(\dot{z}_{x 11}-\dot{z}_{2}\right) \cdot a_{L}+c_{z x 12}\left(\dot{z}_{x 12}-\dot{z}_{2}\right) \cdot a_{L} \\
-k_{z x 11}\left(z_{x 11}-z_{2}\right) \cdot a_{L}+k_{z x 12}\left(z_{x 12}-z_{2}\right) \cdot a_{L}=M_{x} \\
I_{1 \mathrm{y}} \cdot \ddot{\theta}_{\mathrm{y} 1}-c_{z y 11}\left(\dot{z}_{y 11}-\dot{z}_{2}\right) \cdot a_{L}+c_{z y 12}\left(\dot{z}_{y 12}-\dot{z}_{2}\right) \cdot a_{L} \\
-k_{z y 11}\left(z_{y 11}-z_{2}\right) \cdot a_{L}+k_{z y 12}\left(z_{y 12}-z_{2}\right) \cdot a_{L}=M_{y}
\end{array}\right.
$$

where $z_{x 11}, z_{x 12}, z_{y 11}$, and $z_{y 12}$ are the displacements of each cutterhead piece, $z_{1}$ is the axial vibration displacement of the cutterhead, $\theta_{x 1}$ and $\theta_{y 1}$ are the overturning angles of the cutterhead, and $a_{L}$ is the radius of the cutterhead. 
By creating a simultaneous equation from Eqs. (2)(4), the coupling dynamic model of the tunneling and overturning vibrations can be expressed as Eq. (5): diameter is $7930 \mathrm{~mm}$ with 51 cutters, including 8 center, 32 normal, and 11 gauge cutters. The spacing between the center cutters is $101.5 \mathrm{~mm}$ and that between the nor-

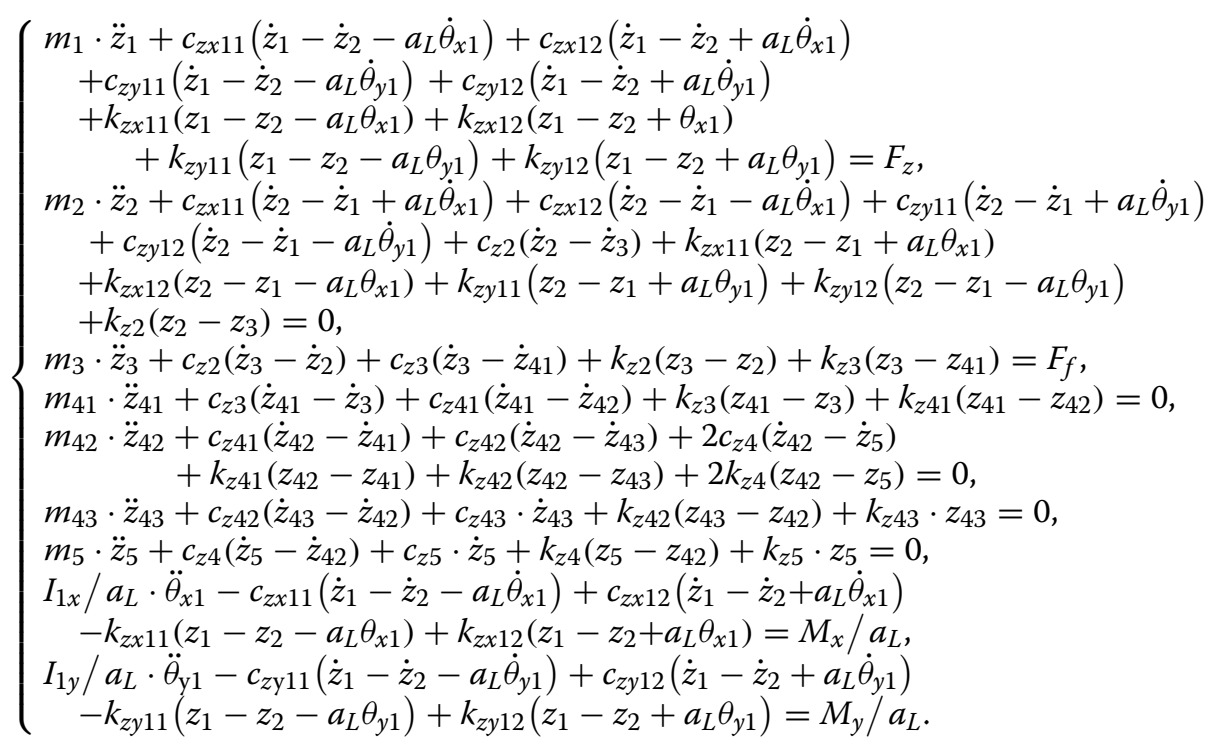

The above vibration transmission equations can be expressed into matrix form as:

$$
\boldsymbol{M} \ddot{X}+\boldsymbol{C} \dot{X}+\boldsymbol{K}=F,
$$

where $X$ is the displacement vector, $M$ is the mass matrix, $K$ is the stiffness matrix, $C$ is the damping matrix, and $F$ is the external excitation.

\section{Solution for Dynamic Parameters}

By considering the TBM used in a water-diversion project as a research object, the layout of the disc cutters in the cutterhead is shown in Figure 4. The cutterhead mal cutters is $83 \mathrm{~mm}$. The inclination angles of the gauge cutters are $65.5^{\circ}, 68.5^{\circ}$, and $70^{\circ}$.

\subsection{External Excitations of the TBM Cutterhead}

The dominant geological formation in the water-diversion project is granite with high compressive strength. Thus, the TBM cutterhead suffers from violent vibrations during the tunneling process. To provide loads for the cutterhead, full-scale rock-breaking tests are performed on a linear cutting machine (LCM) in Central South University [28]. The LCM consists of the frame, moving unit, disc cutter, cutter housing unit, hydraulic unit, control
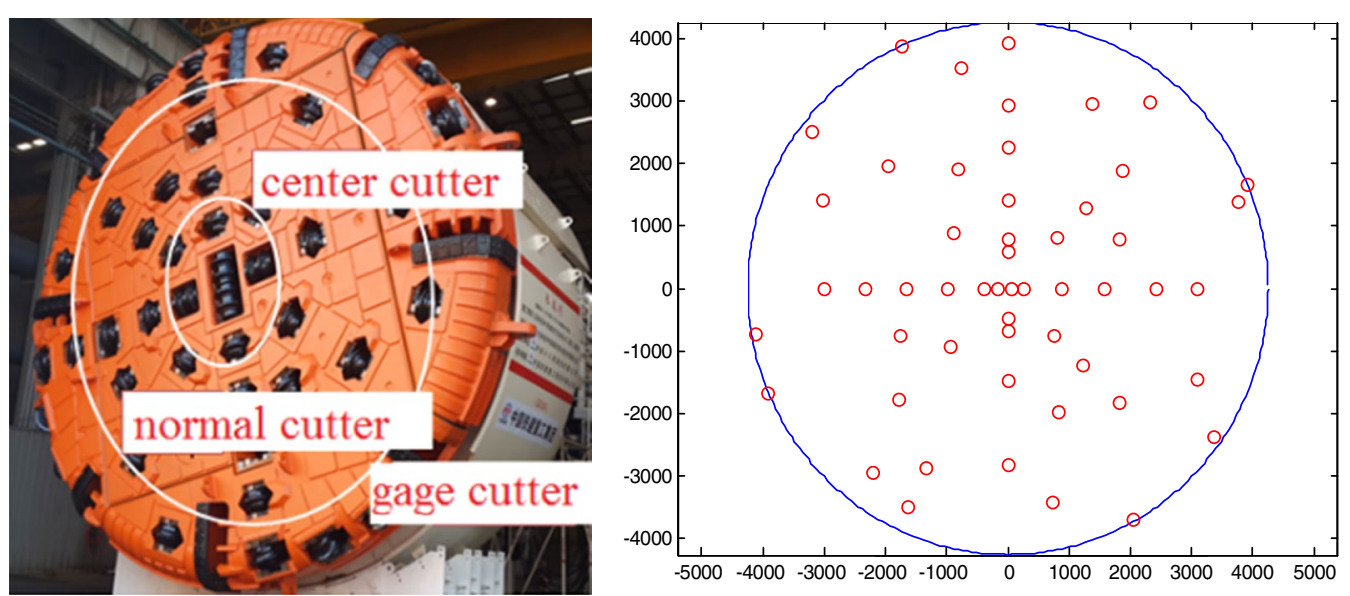

Figure 4 Layout of the disc cutters 


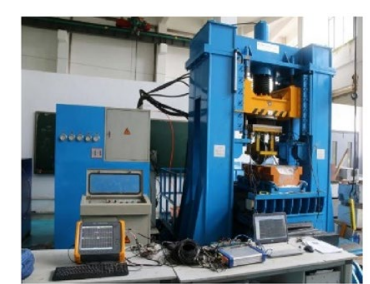

a Linear cutting test platform

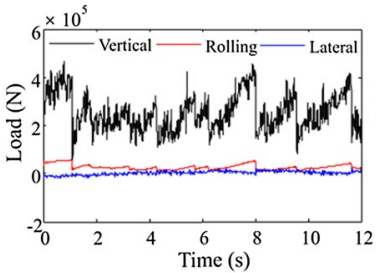

c Normal cutter b Center cutter

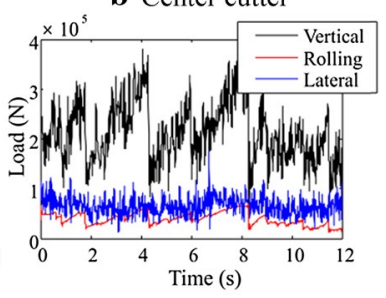

d Gauge cutter



Figure $\mathbf{5}$ Three-dimensional loads of the cutters

unit, and data acquisition unit, as shown in Figure 5. A 17 -in disc cutter is used for the test, and rock samples with dimensions of $900 \mathrm{~mm} \times 380 \mathrm{~mm} \times 280 \mathrm{~mm}$ are used for cutter breaking. The type and mechanical properties of the experimental rock are based on typical TBM geological conditions. By filtering the test data and combining them with simulation, three-dimensional forces can be obtained, as shown in Figure 5. The average vertical and rolling forces in the center cutter are 236 and 34.7 $\mathrm{kN}$, respectively, and the lateral force is concentrated in the range from -80 to $80 \mathrm{kN}$. The average vertical and rolling forces of the normal cutter are 256 and $29.1 \mathrm{kN}$, where the lateral force ranges from -20 to $20 \mathrm{kN}$. The average vertical and rolling forces of the gauge cutter are 214 and $35.74 \mathrm{kN}$, respectively. The lateral force ranges from 20 to $120 \mathrm{kN}$.

Based on the radius, position angle, and tilt angle of the cutter as well as the cutter loads in three directions, the total thrust, total torque and change trend of the radial unbalanced force of the cutterhead can be obtained, which can be presented as time-varying external excitations.

\subsection{Calculation of Equivalent Stiffness and Damping}

Three-row rollers are used in the TBM main bearing, as shown in Figure 6, i.e., main axial rollers that withstand the thrust load, secondary axial roller bearing for the bending moment, and radial rollers that carry the radial load.

The elastic deformation in the elastic line-contact area can be calculated using an empirical equation [29]. The

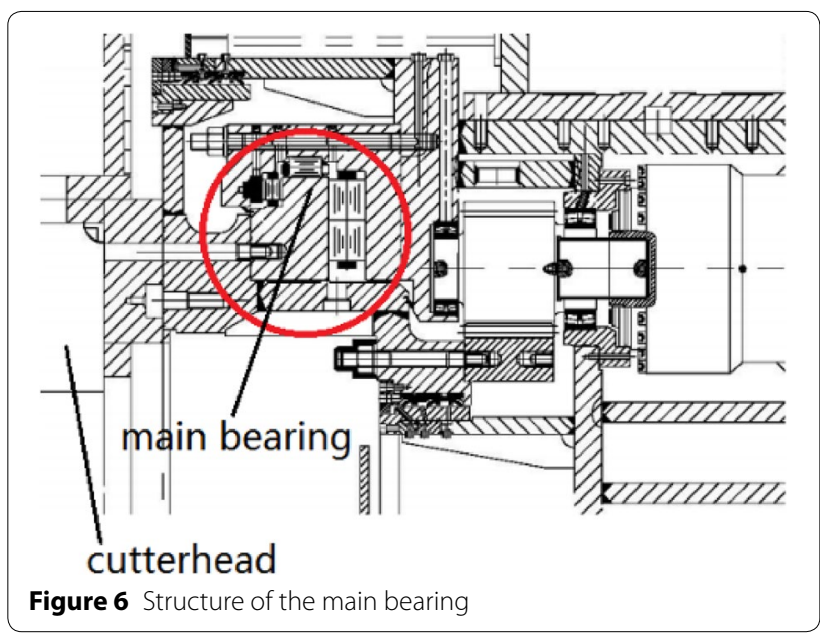

Hertz contact stiffness between the axial roller and raceway is defined as

$$
k_{H}^{\prime}=\frac{d_{Q}}{d_{\delta}}=2.894 \times 10^{5} L_{w e}^{0.8} Q^{0.1},
$$

where $L_{w e}$ represents the effective length of the rollers and $Q$ denotes the contact load.

According to the equation that calculates the oil-film thickness of the contact area, the contact stiffness in the contact area can be expressed as

$$
k_{L}=\frac{d_{Q}}{d_{h_{c}}}=-2.18 \times 10^{3} \frac{E_{0}^{0.03} Q^{1.13}}{\alpha^{0.54}\left(\eta_{0} u\right)^{0.7} R^{0.43} L_{w e}^{0.13}},
$$

where $\alpha$ is the viscosity pressure coefficient of the lubricant, $\eta_{0}$ is the dynamic viscosity of the lubricant, $u$ is the average speed, $R$ is the equivalent curvature radius of the contact area, $E$ is the elastic modulus, and $E_{0}$ is the equivalent elastic modulus.

The contact-area stiffness can be calculated as follows:

$$
k=\frac{1}{1 / k_{H}+1 / k_{L}}
$$

where $k_{H}$ denotes the Hertz contact stiffness and $k_{L}$ denotes the contact stiffness of the oil film.

According to the elastic fluid dynamics, the contact damping between the rollers and roller raceway consists of the roller structural and oil-film damping. Considering the effect of the oil-film thickness, the main-bearing damping coefficient is calculated using the following equation [30].

The damping between the rollers and inner raceway can be expressed as:

$$
c_{1}=\frac{27.4 R^{1.5} \cdot L_{w e}^{0.805} \cdot Z^{0.805} \cdot Q^{0.195}}{\alpha^{0.81} \eta_{0}^{0.05} n_{i}^{1.05} r^{0.645}\left(R_{1}+r\right)^{1.05}(1-\gamma)^{1.695}(1+\gamma)^{1.05} E_{0}^{-0.045}}
$$


The damping between the rollers and surface raceway can be expressed as: tunneling direction are shown in Figure 7. The cutterhead acceleration is concentrated from $-3.5 g$ to $+3.5 g$ with

$$
c_{2}=\frac{27.4 R^{1.5} \cdot L_{w e}^{0.805} \cdot Z^{0.805} \cdot Q^{0.195}}{\alpha^{0.81} \eta_{0}^{0.05} n_{i}^{1.05} r^{0.645}\left(R_{1}+r\right)^{1.05}(1+\gamma)^{1.695}(1-\gamma)^{1.05} E_{0}^{-0.045}},
$$

$$
c=\frac{1}{1 / c_{1}+1 / c_{2}},
$$

where $Z$ is the number of rollers, $n_{i}$ is the rotating speed of the raceway, $r$ is the radius of the roller, and $R_{1}$ is the radius of the inner raceway.

Employing theoretical calculations or experimental measurements to acquire the equivalent stiffness and damping parameters of the cutterhead, main drive, main girder, and support saddle of the gripper shoes is difficult. Therefore, the finite-element method and empirical formula are employed to obtain their stiffness and damping coefficients. The equivalent stiffness of the components can be calculated using Hooke's law. The equivalent damping calculation equation can be expressed as follows:

$$
c=2 \xi \cdot \sqrt{m_{e} \cdot k_{e}},
$$

where $c$ is the structural damping, $\zeta$ is the damping ratio, and $m_{e}$ and $k_{e}$ represent the equivalent mass and equivalent stiffness, respectively.

By summarizing the aforementioned results, the stiffness and damping parameters of each component are obtained, and the partial parameters are listed in Table 1.

\section{Dynamic Responses of TBM Mainframe}

Because the vertical vibration response is similar to the lateral vibration response, this study only analyzes the vibration responses in the lateral, tunneling, and overturning directions of the cutterhead and main girder 1 .

\subsection{Vibration Response in Tunneling Direction}

Under the impact of external excitations, the vibration acceleration curves of the TBM mainframe in the maximum acceleration of more than $7 g$, demonstrating an intense vibration. When the vibration is transmitted to main girder 1 , the acceleration changes to the range from $-2.8 g$ to $2.8 g$ with a peak of $5.9 g$. The frequency responses of the TBM mainframe in the tunneling direction are shown in Figure 8. The vibration energy of the
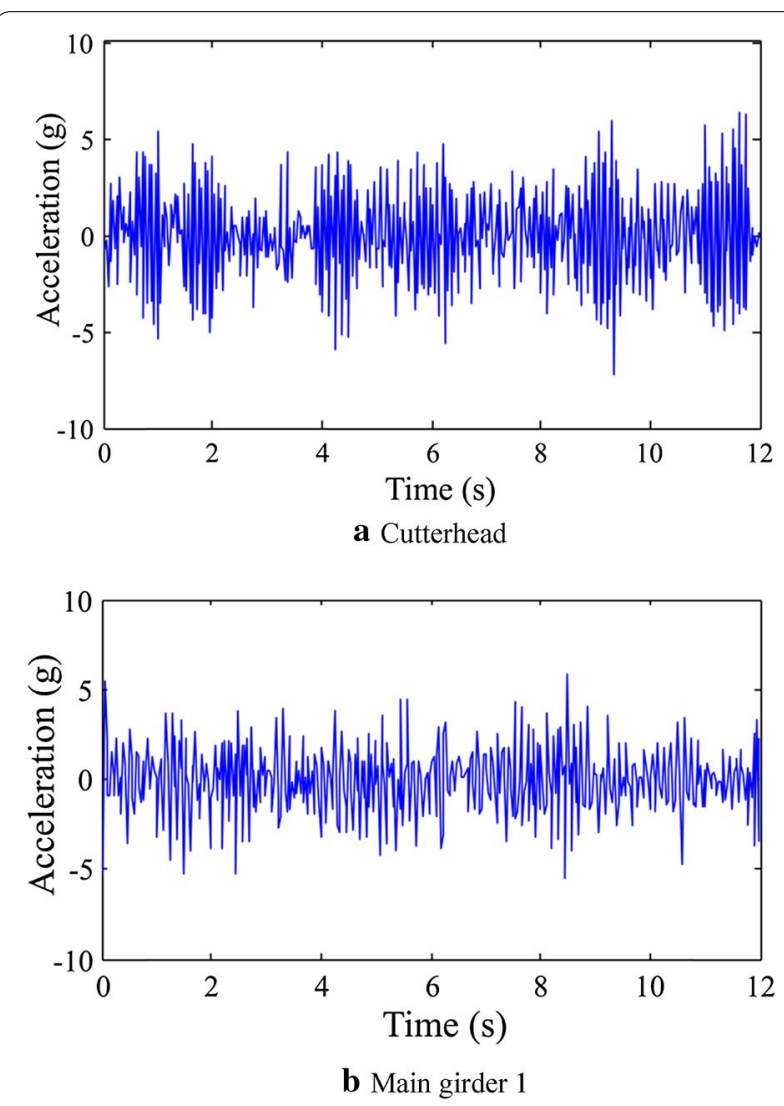

Figure $\mathbf{7}$ Vibration acceleration in the tunneling direction

Table 1 Partial stiffness and damping parameters of mainframe

\begin{tabular}{lllll}
\hline & Cutterhead & Main drive & Main girder 1 & Gripper shoes \\
\hline Lateral stiffness $(\mathrm{N} \mathrm{m})$ & $1.15 \times 10^{10}$ & $5 \times 10^{9}$ & $6.38 \times 10^{8}$ & $8.68 \times 10^{10}$ \\
Overturning stiffness (N m) & $1.56 \times 10^{11}$ & $6.16 \times 10^{10}$ & $5.7 \times 10^{9}$ & $2.11 \times 10^{11}$ \\
Tunneling stiffness (N m) & $4.72 \times 10^{10}$ & $2.5 \times 10^{9}$ & $1.8 \times 10^{10}$ & $3.36 \times 10^{10}$ \\
Lateral damping (N s/m) & $1.75 \times 10^{6}$ & $1.04 \times 10^{6}$ & $1.47 \times 10^{5}$ & $1.84 \times 10^{6}$ \\
Lateral damping (N s/m) & $3.55 \times 10^{6}$ & $7.35 \times 10^{5}$ & $7.9 \times 10^{5}$ & $1.14 \times 10^{6}$ \\
Lateral damping (N s/m) & $4.64 \times 10^{6}$ & $3.91 \times 10^{6}$ & $4.36 \times 10^{5}$ & $5.5 \times 10^{6}$ \\
\hline
\end{tabular}




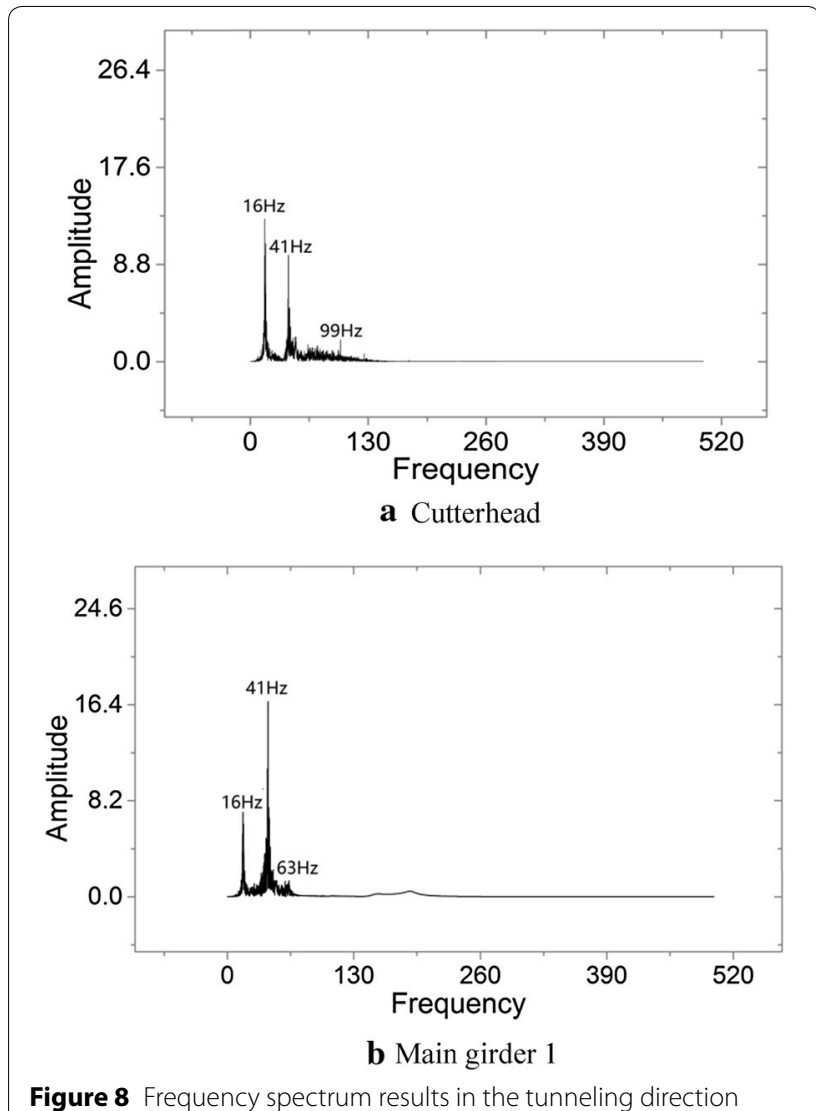

Figure 8 Frequency spectrum results in the tunneling direction

cutterhead is mainly in the frequency range of $0-130$ $\mathrm{Hz}$, and the vibration energy of the main girder is mainly concentrated in the frequency range of $0-70 \mathrm{~Hz}$.

\subsection{Vibration Responses in Overturning Direction}

The vibration angular acceleration of the cutterhead in the overturning direction is shown in Figure 9a, and the overturning angle curve is shown in Figure 9b. The overturning angular acceleration is relatively small, which mainly ranges from -1.5 to $+1.5 \mathrm{rad} / \mathrm{s}^{2}$ with the cutterhead rotation. Overall variation periods of angular acceleration and overturning angle are equal to the period of cutterhead rotation. Frequency responses of the mainframe in the overturning direction are shown in Figure 10 . The vibration energy occurs mainly in the frequency range of $0-130 \mathrm{~Hz}$.

\subsection{Vibration Responses in Lateral Direction}

The lateral acceleration of the cutterhead is shown in Figure 11 , which mainly ranges from $-1.4 g$ to $+1.4 g$ with a maximum of more than $1.45 \mathrm{~g}$. The acceleration of the main girder is slightly lower than that of the cutterhead, which can be attributed to the restraint effect of the bottom support shield. The acceleration of the main girder

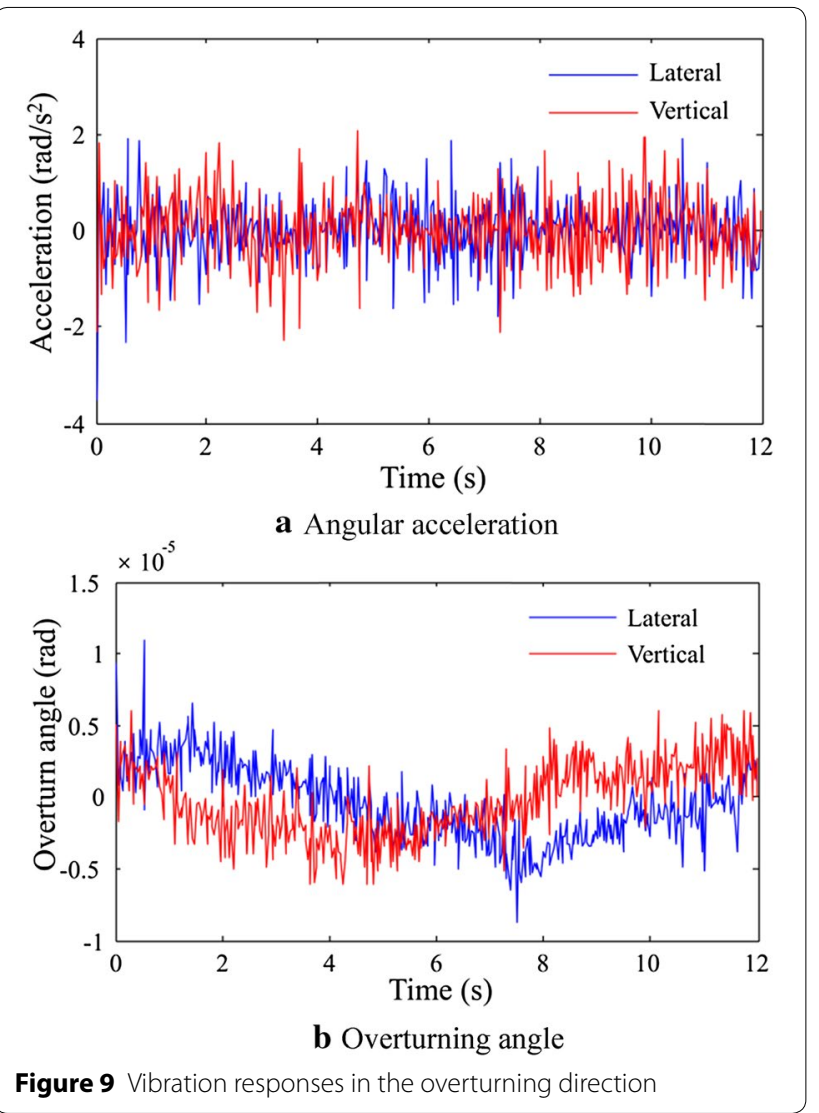

ranges from $-1.0 \mathrm{~g}$ to $+1.0 \mathrm{~g}$ with a maximum of $1.25 \mathrm{~g}$. The frequency spectrum of the lateral vibration is shown in Figure 12. Vibration energy of the cutterhead is distributed in the frequency range of $0-130 \mathrm{~Hz}$, whereas that of the main girder is distributed in the range of $0-100 \mathrm{~Hz}$.

\subsection{Transfer Law of Loads}

The change trend of the propelling resistance transferred from the cutterhead to main girder 1 can be estimated from Figure 13, which clearly shows that the change trends in the transfer process are basically the same. The propelling resistance of the cutterhead is concentrated between 4200 and $22000 \mathrm{kN}$ with an average value of $12848 \mathrm{kN}$. The propelling resistance of the main girder mainly ranges from 2000 to $28000 \mathrm{kN}$ with an average of $12867 \mathrm{kN}$.

From the abovementioned results, the following conclusions can be drawn. Parallel conditions exist between the vibration responses of the cutterhead and main girder. The cutterhead suffers from more intense vibrations with the frequencies concentrated at approximately $0-130 \mathrm{~Hz}$, whereas the frequencies of the main girder vibration are mainly concentrated at approximately $0-100 \mathrm{~Hz}$. The farther the position is from the cutterhead, the lower are 


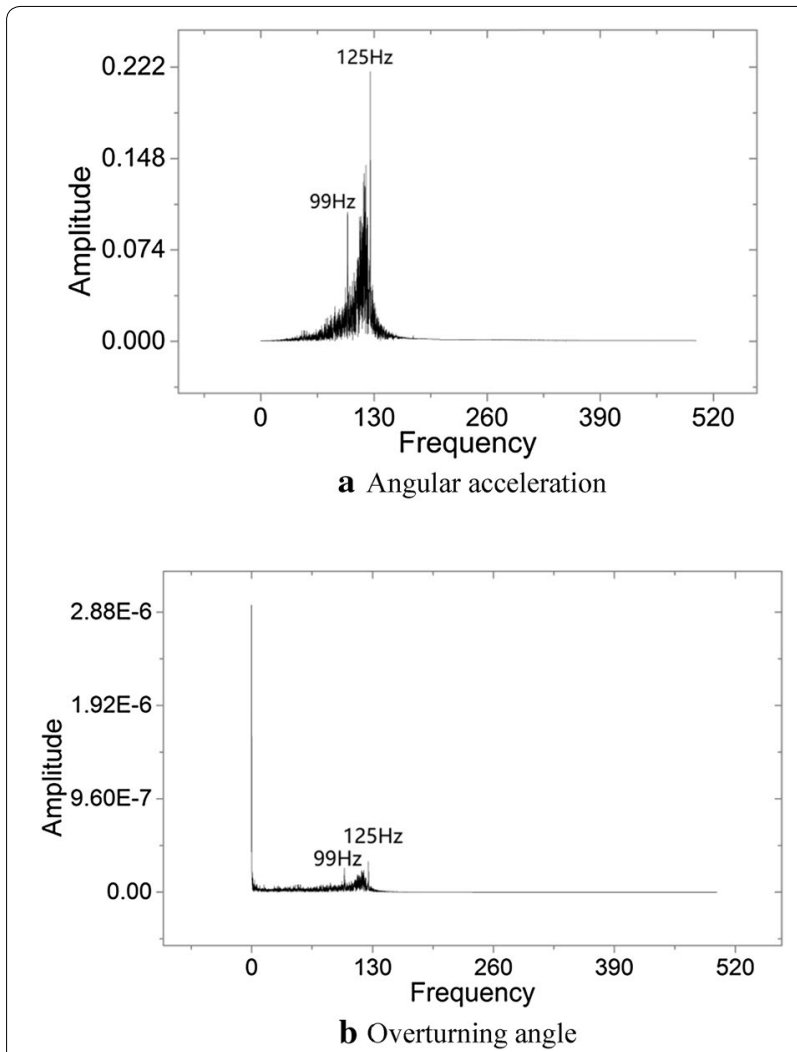

Figure $\mathbf{1 0}$ Frequency spectrum results in the overturning direction

the vibration frequencies. The vibrations in the tunneling direction are more intense than those in the lateral direction. In the load-transfer process, the mean loads remain the same, whereas the change range of the loads expands.

\section{Validation of Dynamic Model Based on Field Tests}

To verify the correctness of the dynamic model, a field test was performed in the TBM mainframe from a waterdiversion project. The vibration tests were conducted using an acceleration test system. The locations of the acceleration test points are shown in Figure 14.

Considering the poor working condition of the cutterhead, acceleration sensors were attached to the manhole of the cutterhead, i.e., point A in Figure 14. The test points in the main drive were located around the $\mathrm{B}$ point on the main girder connecting plate, and the test points for the main girder were located on the $C, D$, and E point positions. The vibration signals in the manhole were transferred in a wireless manner, whereas those of the other test points were directly obtained through the transmission lines. At each test point, three acceleration sensors for the lateral, vertical, and tunneling directions were installed.
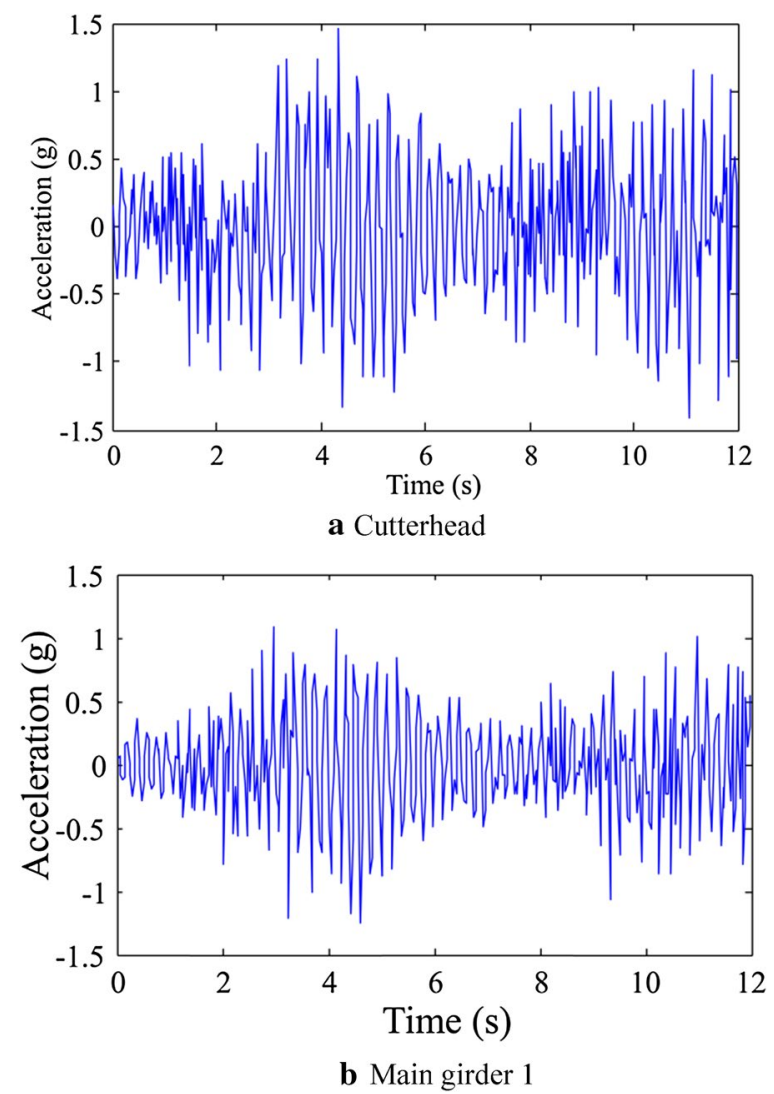

Figure 11 Vibration acceleration in the lateral direction

\subsection{Vibration Test of Cutterhead}

The field-test values of the vibration of the cutterhead are compared with the theoretical values, as shown in Figure 15. The measured values of the cutterhead lateral acceleration are larger than those calculated by the model, which mainly range from $-2 g$ to $+2 g$. The meansquare deviation of the lateral-test acceleration is $1.01 \mathrm{~g}$, whereas the calculated value is $0.61 g$. The measured values of the vibration acceleration in the tunneling direction are lower than the calculated values, which mainly range from $-3 g$ to $+3 g$. The mean-square deviation of the measured tunneling acceleration is $1.92 g$, and that of the calculated acceleration is $2.1 g$.

\subsection{Vibration Test of Main Girder}

The measured and calculated acceleration values of the main girder are shown in Figure 16, which shows that the measured values of the lateral-vibration acceleration in the main girder are larger than the calculated values, mainly ranging from $-2 g$ to $+2 g$. The meansquare deviation of the lateral measured acceleration is $0.86 \mathrm{~g}$, and that of the calculated value is $0.52 \mathrm{~g}$. In the tunneling direction, the test values of the vibration 


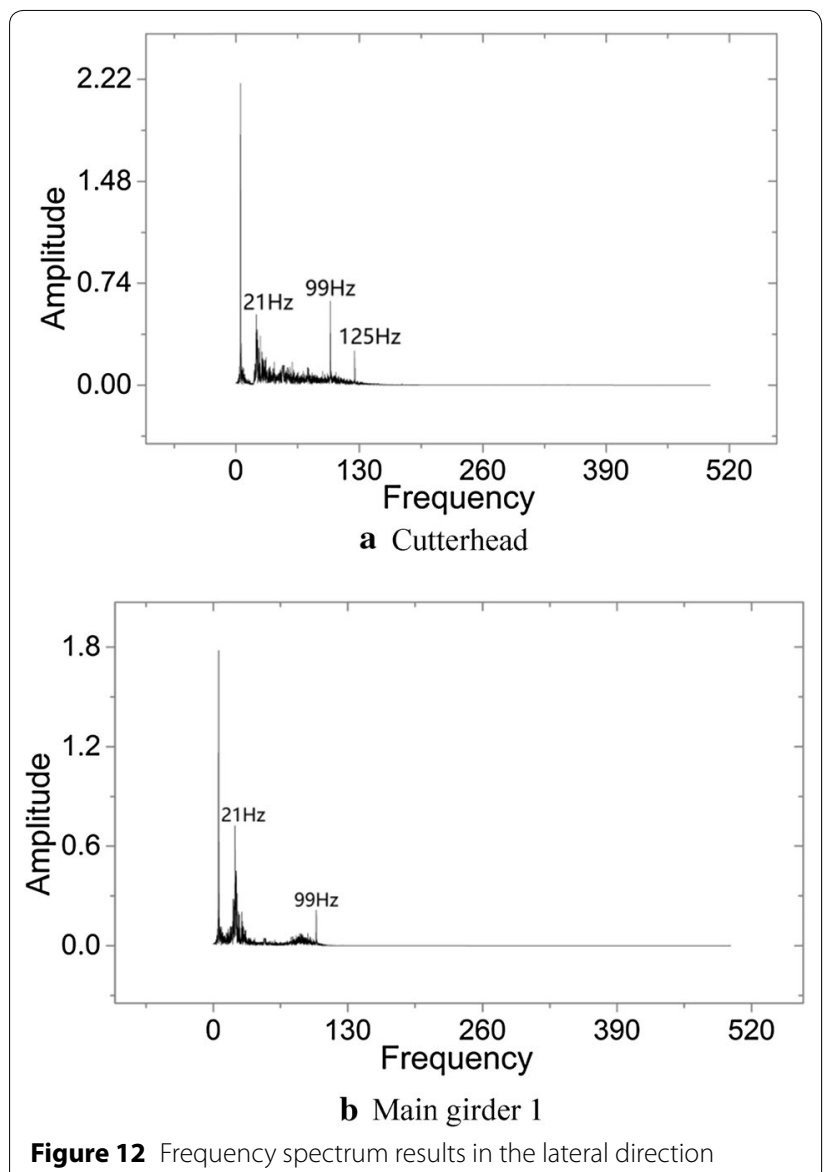

Figure 12 Frequency spectrum results in the lateral direction

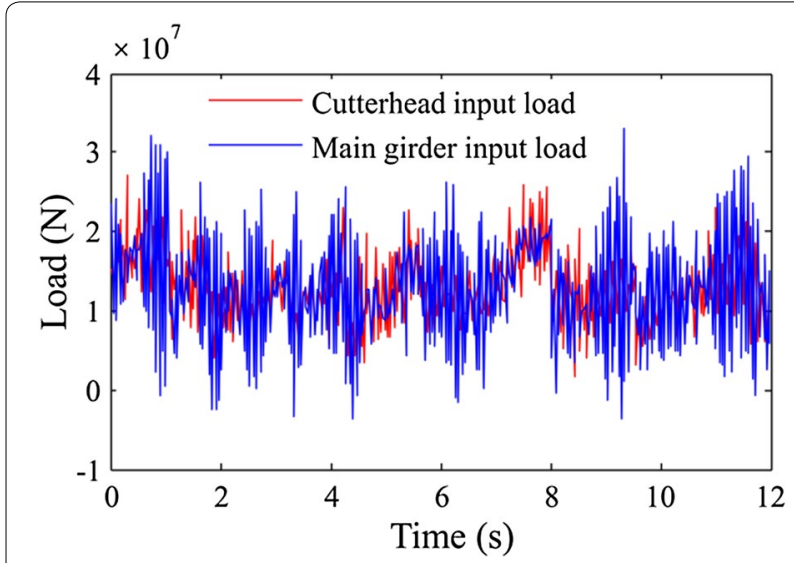

Figure 13 Propelling resistance transmission

acceleration are lower than the calculated values, which mainly range between $-2.3 g$ and $2.3 g$ with the maximum value reaching $5 g$. Mean-square deviation of the measured tunneling acceleration is $1.51 \mathrm{~g}$, and that of the calculated acceleration is $1.72 g$.



Figure 14 Locations of the acceleration sensors

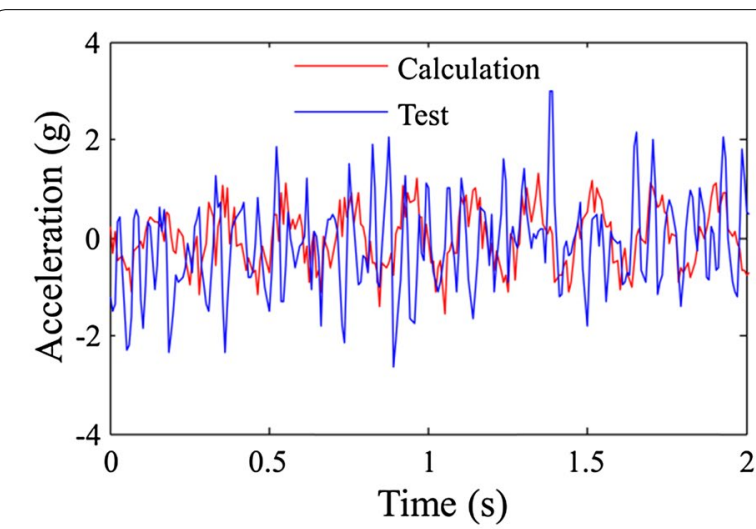

a Lateral direction

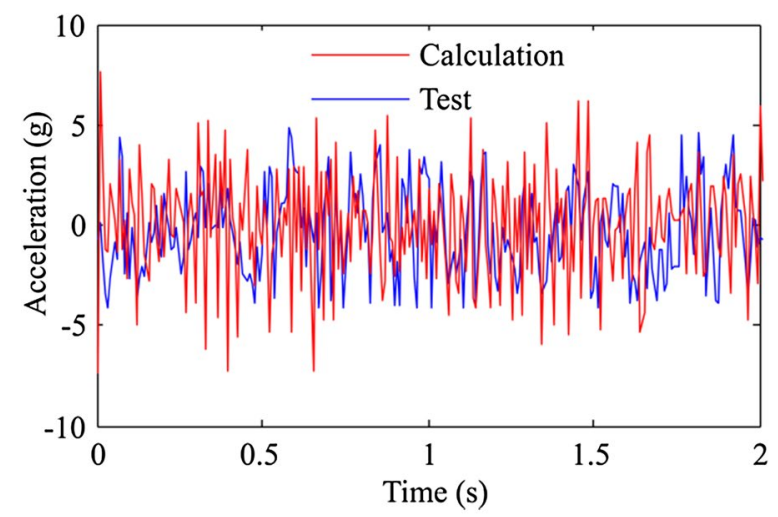

b Tunneling direction

Figure 15 Measured and calculated accelerations of cutterhead

Comparison of the field-test and calculated values of the acceleration in the TBM mainframe reveals that the calculated values of the lateral and vertical vibration 

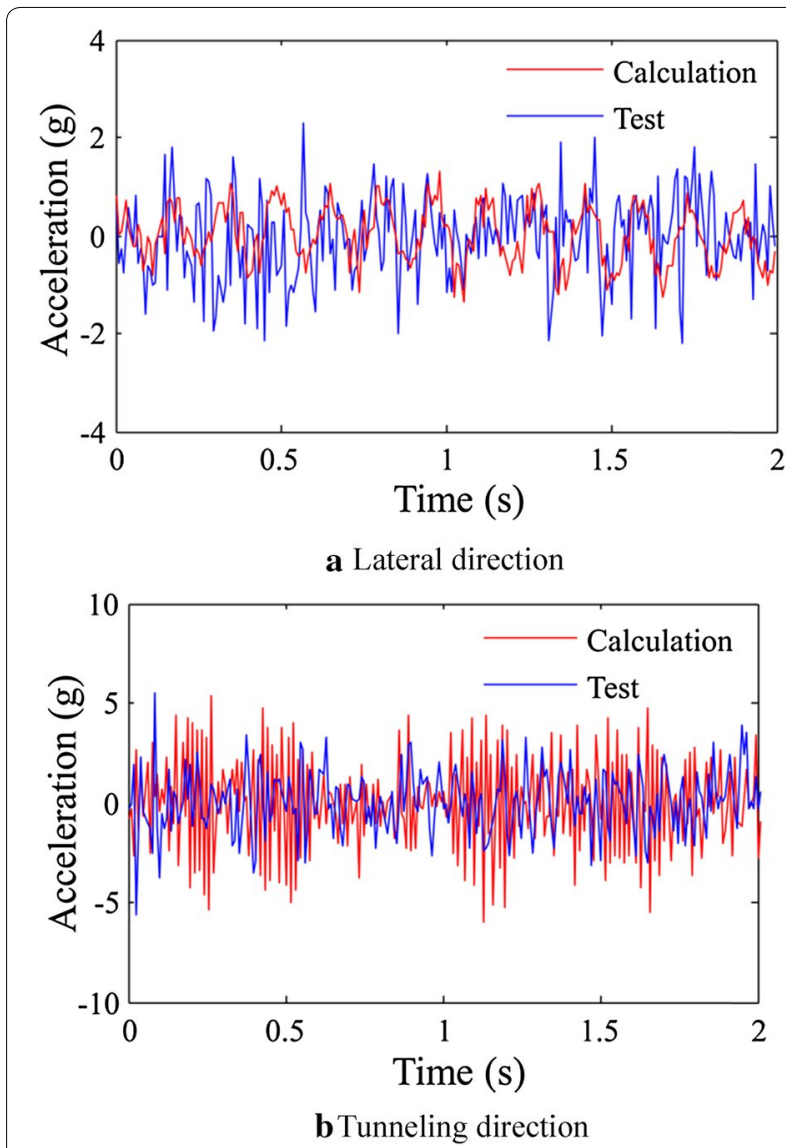

Figure 16 Measured and calculated accelerations of main girder

acceleration are lower than the test values, whereas the calculated values of the vibration acceleration in the tunneling direction is larger than the measured values. The reasons for the differences in the tunneling and other directions can be attributed to the complex internal structure of each component in the mainframe and the slant stiffened plate of the cutterhead. In the transmission process, partial vibrations in the tunneling direction can be transmuted into other vibrations such as the lateral and vertical vibrations due to the slant stiffened plate and complex internal structure of the mainframe.

The calculated and measured acceleration values of the TBM mainframe have the same magnitude within a relatively acceptable error range. The major error comes from the simplification of the complex internal structure of the TBM mainframe coupled with errors in the calculations of the equivalent stiffness and damping.

\section{Conclusions}

In this study, a dynamic model that includes the cutterhead, main drive, main girder, and supportthrust system is established. The dynamic response characteristics of the TBM mainframe and the transfer trend of loads are investigated both theoretically and by field tests. The main results are summarized as follows.

(1) In the tunneling process, the lateral, vertical, and tunneling vibrations of the TBM mainframe have the same vibration level in which the changes are similar to those of the external excitations.

(2) Parallel conditions exist between the vibration responses of the cutterhead and main girder. The multi-directional vibration of the cutterhead is more intense than that of the main drive, main bearing, and main girder. The vibrations in the tunneling direction are more intense than those in the lateral direction. The frequencies of the cutterhead vibration energy are predominantly concentrated at approximately $0-130 \mathrm{~Hz}$, and those of the main girder vibration energy are mainly concentrated at approximately $0-100 \mathrm{~Hz}$. As the distance from the cutterhead increases, the vibration energy is concentrated in a lower frequency. During the design of the TBM mainframe, measurement of the vibration reduction needs to be considered to prevent fatigue damage and breakdown of the cutterhead and damage to the main bearing.

(3) In the process of mainframe load transfer from the tunneling direction, the average load and change trend of the loads remain constant, whereas the range of the loads expands by 1.4 times with the increase in the load amplitude. As a result, when the strength of the components in the back end of the mainframe is designed and checked, the external loads from the cutterhead need to be amplified.

(4) The lateral measured acceleration of the TBM mainframe is concentrated in the range from $-2 g$ to $+2 g$, and the measured acceleration in the tunneling direction is mainly concentrated in the range from $-3 g$ to $+3 g$. Comparison of the theoretical values with the actual test values from three directions in the mainframe reveals that the measured and calculated vibrations are basically the same within a relatively acceptable range of error. The results have verified the correctness of the modeling and the solution of the dynamic model, which confirm the weakest components of TBM mainframe and provide a theoretical basis for the design and evaluation of the mainframe as well as the external loads for mainframe fatigue-life analysis.

Authors' Contributions

$L L$ and $Y X$ were in charge of the whole research; $L L$ analyzed results and wrote the manuscript; ZL assisted with sampling and field test. CW, YC and QT 
assisted with modeling, data analyzing and manuscript writing. All authors read and approved the final manuscript.

\section{Authors' Information}

Laikuang Lin, born in 1988, is currently a postdoc at College of Mechanical and Electrical Engineering, Central South University, China. He received his PhD degree from Central South University, China, in 2017. His research interests include dynamics, and design theory of TBM cutterhead.

Yimin Xia, born in 1967, is currently a professor at College of Mechanical and Electrical Engineering, Central South University, China. He received his PhD degree from Central South University, China, in 2006. His research interests include hydraulic transmission and control, mechanical engineering.

Zhengguang Li, born in 1994, received his master degree from Central South University, China, in 2018. His current research interests include dynamics, transmission and control technology.

Caizhang Wu, born in 1990, received his master degree from Central South University, China, in 2016. His research interests include dynamics and mechanical design.

Yongliang Cheng, born in 1978, is currently a PhD candidate at Central South University, China. His research interests include design theory of TBM.

Qing Tan, born in 1955, is currently a professor at Central South University, China. His research interests include dynamics, and rock breaking mechanism of disc cutters.

\section{Competing Interests}

The authors declare that they have no competing interests.

\section{Funding}

Supported by National Key R\&D Program of China (Grant No. 2017YFB1302603), National Natural Science Foundation of China (Grant No. 51905550), National Basic Research Program of China (Grant No. 2013CB035401), and China Postdoctoral Science Foundation (Grant No. 2019M652795).

\section{Author Details}

${ }^{1}$ College of Mechanical and Electrical Engineering, Central South University, Changsha 410083, China. ${ }^{2}$ State Key Laboratory of High Performance Complex Manufacturing, Central South University, Changsha 410083, China.

${ }^{3}$ China Railway Construction Heavy Industry Co., Ltd., Changsha 410100, China.

Received: 20 January 2019 Accepted: 15 November 2019 Published online: 03 December 2019

\section{References}

[1] J Q Liu, J B Ren, W Guo. Thrust and torque characteristics based on a new cutter-head load model. Chinese Journal of Mechanical Engineering, 2015, 28(4): 801-809.

[2] H B Xie, Z B Liu, H Y Yang. Pressure regulation for earth pressure balance control on shield tunneling machine by using adaptive robust control. Chinese Journal of Mechanical Engineering, 2016, 29(3): 598-606.

[3] SY Zhou, Y L Kang, CX Su, et al. Prediction of thrust force requirements for TBMs based on mechanical analysis. Journal of Mechanical Engineering, 2016, 52(20): 76-82. (in Chinese)

[4] Y M Xia, L K Lin, DWu, et al. Geological adaptability matching design of disc cutter using multicriteria decision making approaches. Journal of Central South University, 2018, 25(4): 843-854.

[5] J Rostami, S H Chang. A closer look at the design of cutterheads for hard rock tunnel-boring machines. Engineering, 2017, 3(6): 892-904.

[6] Y M Xia, Y C Tian, Q Tan, et al. Side force formation mechanism and change law of TBM center cutter. Journal of Central South University, 2016, 23(5): 1115-1122.

[7] J Z Huo, W Z Wang, W Sun, et al. The multi-stage rock fragmentation load prediction model of tunnel boring machine cutter group based on dense core theory. The International Journal of Advanced Manufacturing Technology, 2017, 90(1-4): 277-289.
[8] H Shi, HYYang, G F Gong, et al. Determination of the cutterhead torque for EPB shield tunneling machine. Automation in Construction, 2011, 20(8): 1087-1095.

[9] SY Zhou, Y L Kang, H M Xie, et al. An approach integrating dimensional analysis and field data for predicting the load on tunneling machine. KSCE Journal of Civil Engineering, 2019, 23(7): 3180-3187.

[10] Q Geng, ZY Wei, H Meng, et al. Mechanical performance of TBM cutterhead in mixed rock ground conditions. Tunnelling and Underground Space Technology, 2016, 57: 76-84.

[11] Y M Xia, C ZWu, H Lan, et al. Mechanical performance analysis and comparison of typical TBM cutterhead. Journal of Harbin Engineering University, 2016 37(08): 1136-1142. (in Chinese)

[12] Y Zhao, Q M Gong, ZYTian, et al. Torque fluctuation analysis and penetration prediction of EPB TBM in rock-soil interface mixed ground. Tunnelling and Underground Space Technology, 2019, 91: 103002.

[13] M Entacher, G Winter, T Bumerger, et al. Cutter force measurement on tunnel boring machines - System design. Tunnelling and Underground Space Technology, 2012, 31(5): 97-106.

[14] M Entacher, G Winter, R Galler. Cutter force measurement on tunnel boring machines - Implementation at Koralm tunnel. Tunnelling and Underground Space Technology, 2013, 38(3): 487-496.

[15] Q Geng, ZYWei, H Meng. An experimental research on the rock cutting process of the gage cutters for rock tunnel boring machine (TBM). Tunnelling and Underground Space Technology, 2016, 52: 182-191.

[16] Q Zhang, CY Qu, ZX Cai, et al. Modeling of the thrust and torque acting on shield machines during tunneling. Automation in Construction, 2014, 40(4): 60-67.

[17] Q Zhang, Z D Hou, GY Huang, et al. Mechanical characterization of the load distribution on the cutterhead-ground interface of shield tunneling machines. Tunnelling and Underground Space Technology, 2015, 47: 106-113.

[18] W Sun, J X Ling, J Z Huo, et al. Dynamic characteristics study with multidegree-of-freedom coupling in TBM cutterhead system based on complex factors. Mathematical Problems in Engineering, 2013, 2013(3): 657-675.

[19] J X Ling, W Sun, J Z Huo, et al. Study of TBM cutterhead fatigue crack propagation life based on multi-degree of freedom coupling system dynamics. Computers and Industrial Engineering, 2015, 83: 1-14.

[20] X H Li, H B Yu, M Z Yuan, et al. Dynamic modeling and analysis of shield TBM cutterhead driving system. Journal of Dynamic Systems, Measurement, and Control, 2010, 132(4):1-14.

[21] J Z Huo, X L Sun, G Q Li, et al. Multi-degree-of-freedom coupling dynamic characteristic of TBM disc cutter under shock excitation. Journal of Central South University, 2015, 22(9): 3326-3337.

[22] J Z Huo, N Hou, W Sun, et al. Analyses of dynamic characteristics and structure optimization of tunnel boring machine cutter system with multi-joint surface. Nonlinear Dynamics, 2017, 87(1): 237-254.

[23] J Z Huo, HY Wu, W Sun, et al. Electromechanical coupling dynamics of TBM main drive system. Nonlinear Dynamics, 2017, 90(4): 2687-2710.

[24] HYWu, J Z Huo, Z C Meng, et al. Load characteristics study with a multicoupling dynamic model for TBM supporting system based on a field strain test. Tunnelling and Underground Space Technology, 2019, 91: 103016.

[25] D Festa, W Broere, JW Bosch. Kinematic behaviour of a Tunnel Boring Machine in soft soil: Theory and observations. Tunnelling and Underground Space Technology, 2015, 49: 208-217.

[26] K Z Zhang, H D Yu, XX Zeng, et al. Numerical simulation of instability conditions in multiple pinion drives. Proceedings of the Institution of Mechanical Engineers, Part C: Journal of Mechanical Engineering Science, 2011, 225(6): 1319-1327.

[27] T Huang, X L Wang, H T Liu, et al. Force analysis of an open TBM grippingthrusting-regripping mechanism. Mechanism and Machine Theory, 2016, 98 101-113.

[28] L K Lin, Q S Mao, Y M Xia, et al. Experimental study of specific matching characteristics of tunnel boring machine cutter ring properties and rock Wear, 2017, 378: 1-10.

[29] Y M Xia, C Qian, Z G Li, et al. Vibration characteristics of TBM supportingthrusting system. Journal of Zhejiang University (Engineering Science), 2018, 52(2): 233-239. (in Chinese)

[30] P Dietl, J Wensing, G C V Nijen. Rolling bearing damping for dynamic analysis of multi-body systems - Experimental and theoretical results. Proceedings of the Institution of Mechanical Engineers Part K Journal of Multi-body Dynamics, 2000, 214(1): 33-43. 